\title{
Estimating the benefits of introducing a rapid STI service for Symptomatic men using time-driven activity-based costing data
}

Horner $\mathrm{P}^{1,2}$, Crofts $\mathrm{M}^{2}$, North $\mathrm{P}^{3}$, Steer $\mathrm{J}^{3}$, Turner $\mathrm{J}^{3}$, Wheeler $\mathrm{H}^{2}$, Hartman $\mathrm{C}^{2}$, Warr $\mathrm{L}^{2}$, Muir $\mathrm{P}^{3}$

${ }^{1}$ University of Bristol, ${ }^{2}$ Unity Sexual Health Centre, ${ }^{3}$ Public Health England South West Regional Laboratory, Bristol, United Kingdom

Background: Sexual health services are under increasing pressure to improve the patient experience, yet reduce costs. Antimicrobial resistance (AMR) to macrolides has emerged rapidly in Mycoplasma genitalium (MG) over the last 10 years due to the widespread use of azithromycin $1 \mathrm{~g}$ and the failure to undertake a test of cure following treatment, and quinolone resistance is beginning to emerge..$^{1,2}$ It has recently been demonstrated that detecting MG at presentation in men with NGU and undertaking macrolide AMR testing improves outcomes and reduces the risk of AMR developing. ${ }^{3}$ However including MG NAAT testing in men with NGU would increase costs. We had previously demonstrated how rapid CT and NG NAAT testing could improve outcomes for men with symptoms and developed a costing tool Healthcare Optimisation Planner which uses a time-driven activity-based costing (TDABC) modelling ${ }^{4-6}$ approach, to support a business case to introduce the Hologic Panther to the Unity Sexual Health Clinic which can provide a CT NG and MG NAAT result from a patient self-test in 4 hours. Patients with symptoms would be asked to self test and re-attend to see a clinician in 4 hours, who would have access to the CT,NG and MG NAAT test result. This service will be introduced for both males and females, autumn 2018, at Unity Sexual Health and will be evaluated by the National Institute for Health Research Collaboration for Leadership in Applied Health Research and Care West (NIHR CLAHRC West. (see poster on estimating the benefits for women)

Methods: Unity Sexual Health manages approximately 2000 men per annum with symptoms of urethritis. A multidisciplinary approach to care is delivered through a mixture of doctors in training and nurses supported by a senior clinician. A TDABC model ${ }^{4-6}$ for all symptomatic men attending our clinic was developed using electronic patient record (EPR) data from 422 consecutive male patients with symptoms of urethritis (discharge, dysuria, penile irritation and testicular pain) over a 3 month period and used to develop a care pathway model for men with urethritis. Our current model (Figure) consists of activities a male symptomatic patient may experience and the transition probabilities of a client through the pathway between these activities. An activity consists of the associated 'Staff' and 'Consumables' costs which were based on recent estimates from the literature ${ }^{5,7}$ and time taken was estimated using data on the number of patients seen per clinical session. For simplicity only nurse band $6 / 7$ were considered as the clinician with a staff cost per minute(£0.89) which included indirect and overhead costs. Patient time and associated costs were excluded. The cost of chlamydia (CT) /gonorrhoea (NG), and Mycoplasma genitalium (MG) NAATs were assumed to be $£ 12.51 \& £ 7.62$ and NG culture £7.55 respectively.

The effect on the care pathway after the introduction of rapid STI testing service with the Hologic Panther was based on expert review of the literature with the number of men with CT and NG remaining the same. The prevalence of MG +ve NGU was assumed to be $23 \% 36 / 157$ ), of these $40 \%$ (14) would be macrolide resistant. Men with NGU are currently treated with doxycycline $100 \mathrm{mgs}$ bd 7 days which would also be used in the new rapid service for men with pathogen negative NGU (>= 5 PMNLs/hpf) and chlamydia, for MG +ve men doxycycline $100 \mathrm{mgs}$ bd 7 days then azithromycin $1 \mathrm{~g}$ plus $250 \mathrm{mgs}$ od 4 days. It was assumed this regimen would cure $40 \%$ of men with macrolide AMR. The model did not incorporate MG AMR testing. Reduced re-attendance (20\%->5\%) for CT +ve men would happen because of improved treatment compliance with no sexual intercourse until treatment is completed and partner(s) treated. The cost of providing care for 2,000 men was then estimated with Healthcare Optimisation Planner summating the costs of all the activities.

Results: For symptomatic men it was estimated there would be $36 \%$ fewer urethral smears and the number of follow-up visits would be reduced by $78 \%$ (719->157) with a $3.5 \%$ reduction in overall costs from $£ 222000$ to $£ 214000$ with staff costs reduced by $3.9 \%$ and diagnostic costs by approximately the same. Although less nursing time would be required to manage patients as result of a reduced number of follow-up visits this would be partially off-set by an increase in health adviser time for partner notification at time of initial consultation.

Conclusion: The introduction of a rapid STI service is likely to be cost effective and improve the patient experience with early knowledge of their results, fewer invasive tests and less follow-up visits as a result of receiving the correct initial treatment. This is also likely to improve partner notification rates and reduce the use of unnecessary antibiotics.

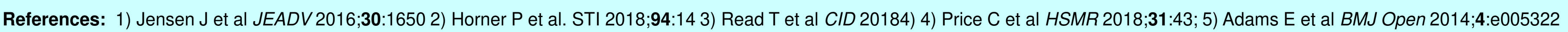
6) Horner et al ISSTDR Brisbane 2015 7) Nicholls J et al. STI 2018;94:93b



Figure : TBACD models of the current Unity male urethritis care pathway and a new rapid pathway incorporating rapid CT NG and MG NAAT testing (see methods). Boxes represent "activities" with patient numbers based on review of 422 EPRs and numbers in bold represent probability of next activity happening. 